\title{
Profile of Obstetric Patients in Intensive Care Unit and Untold Stories behind Maternal Deaths and Life-threatening Complications
}

\author{
Susruta Bandyopadhyay \\ Indian Journal of Critical Care Medicine (2021): 10.5005/jp-journals-10071-23798
}

Obstetric patients who require intensive care unit (ICU) care, take a small share of total ICU admissions. Divatia et al. in their multicenter point prevalence study, which looked into the case-mix of 120 Indian ICUs, reported just 35 obstetric patients out of total 4,209 patients. This makes up to $0.8 \%$. The average APACHE II score of these patients was $13.7 \pm 8.5$ and there were only one ICU nonsurvivor and one hospital nonsurvivor.' This percentage varies across regions and ICUs. It is higher in developing countries than in developed countries. Vasquez et al. in Argentina, when studying obstetric patients in ICU, found as high as $10 \%$ of ICU admissions to be obstetric patients. They also had higher mortality. ${ }^{2}$

Obstetric critical care stands out from the other branches of critical care. There remains the question of not only the patient's outcome but also the fetal outcome. There are diseases specifically due to obstetric complications like antepartum and postpartum hemorrhages and the whole gamut of the diseases related to pregnancy-induced hypertension (PIH). Again there are usual diseases of the ICU, which when occurring in a pregnant patient, are modified due to the different physiologies of pregnancy. One may surmise that the maternal mortality rate, although depending largely on antenatal care and obstetric facilities, also depends on good obstetric critical care. India still has a high maternal mortality rate, although it has steadily improved in the last 5 years. ${ }^{3}$

With this background, the present issue of the Indian Journal of Critical Care Medicine publishes a retrospective case series from the dedicated obstetric ICU in a tertiary care teaching hospital. The patients admitted in the ICU over 18 months were studied. The authors admitted that, as there was also a medical ICU in their hospital, not all obstetric patients, who required ICU care were admitted in their obstetric ICU. ${ }^{4}$

There has been studies to this effect from time to time, both prospective and retrospective. They have been from different regions, single-centered and multicentered, from the developed world and the developing world. As one goes through them, one definitely can make out some patterns. The patients in the developed world tend to be older and suffer more from the complications of PIH than from hemorrhage. The reverse is usually true for the patients from the developing world.

The present study includes all patients admitted in this ICU during pregnancy and up to 6 weeks after the delivery. The age of the patients were younger compared to western countries and the majority were multigravida. The authors intentionally did not
Department of Critical Care, AMRI Hospitals, Kolkata, West Bengal, India

Corresponding Author: Susruta Bandyopadhyay, Department of Critical Care, AMRI Hospitals, Kolkata, West Bengal, India, e-mail: subandyo@gmail.com

How to cite this article: Bandyopadhyay, S. Profile of Obstetric Patients in Intensive Care Unit and Untold Stories behind Maternal Deaths and Life-threatening Complications. Indian J Crit Care Med 2021;25(4): 362-363.

Source of support: Nil

Conflict of interest: None

use any disease severity scores in these patients. The effect is that one gets the idea of the diagnoses with which the patients were admitted. But one does not get an idea of the severity of their illness. The authors justify their action, by citing the most of the current severity scores overestimate the mortality in pregnant women. This has been definitely validated by several studies. For example, RojasSuarez et al. showed that APACHE II \& SAPS II both overestimated mortality in 726 obstetric critical care patients. ${ }^{5}$ However we find that a few Indian as well as western studies do mention such scores, which give us a general idea about the seriousness of the patient's condition.

We note that the major cause of ICU admission remains major obstetric hemorrhages ( $\mathrm{MOH}$ ) in Asian countries. In the present trial, $47.5 \%$ of the admissions were due to obstetric hemorrhages. The second biggest group was that of the patients having complications of PIH, they made up $35.64 \%$ of the patients. A similar picture was found in some other studies from India. A study from a tertiary care teaching hospital in Pondicherry reported that $51 \%$ admissions in the ICU for the obstetric patients were due to obstetric hemorrhage, and just $18 \%$ due to the complications of PIH. ${ }^{6}$ Similarly a 12 -year study in a tertiary care hospital from Saudi Arabia showed $32.8 \%$ admissions due to obstetric hemorrhage and just $17.2 \%$ for $\mathrm{PIH}^{7}$ This contrasts sharply with a study from Argentina where 161 patients of obstetric critical care were studied. PIH and related complications comprised $40 \%$ of the patient load and $\mathrm{MOH}$ made up just $16 \% .^{2}$

Another bane of the ICUs in the developing world, namely, sepsis was quite low in number in this study. Just 5.94\% patients had sepsis. Compare this to $28.2 \%$ incidence of sepsis in 104 ICU admissions

(0) Jaypee Brothers Medical Publishers. 2021 Open Access This article is distributed under the terms of the Creative Commons Attribution 4.0 International License (https://creativecommons.org/licenses/by-nc/4.0/), which permits unrestricted use, distribution, and non-commercial reproduction in any medium, provided you give appropriate credit to the original author(s) and the source, provide a link to the Creative Commons license, and indicate if changes were made. The Creative Commons Public Domain Dedication waiver (http://creativecommons.org/publicdomain/zero/1.0/) applies to the data made available in this article, unless otherwise stated. 
due to obstetric complications in a tertiary care hospital in NOIDA. ${ }^{8}$ Even a nationwide observational study from the Netherlands in 98 obstetric ICUs showed the incidence of sepsis to be higher at $6.6 \% .{ }^{9}$

Interestingly none of the trials other than the trial from Netherland mentions venous thromboembolisms (VTEs). The Dutch trial mentions fatality, albeit small in number, from VTEs. Do the warmer countries truly have less VTEs or are they missing the diagnoses, remains the question.

Although many of the obstetric patients requiring ICU care required mechanical ventilation, some required renal replacement therapy, and a few were in shock, the mortality remained low across the studies. Our present study showed crude mortality of $7.89 \%$, which was lower than the Pondicherry series (13\%), Argentina series (11\%) but higher than the Dutch series (3.5\%). The authors attribute this achievement to their better antenatal care, and the availability of a blood bank in their hospital, which facilitated the management of hemorrhagic complications. In fact they have stated that the mortality was higher among patients who were irregular with their antenatal checkups. Although the numbers were not mentioned.

This study along with other similar studies depicts patterns on which one can make conjectures about better handling of critical obstetric patients. The ultimate goal remains to cut down maternal mortality to as low as possible.

WHO has said, "There is a story behind every maternal death or life-threatening complications." We look for such untold stories.

\section{OrCID}

Susruta Bandopadhyay (1) https://orcid.org/0000-0001-5732-5461

\section{References}

1. Divatia JV, Amin PR, Ramakrishnan N, Kapadia FN, Todi S, Sahu S, et al. Intensive care in India: the Indian intensive care case mix and practice patterns study. Indian J Crit Care Med 2016;20(4):216-225. DOI: 10.4103/0972-5229.180042.

2. Vasquez DN, Estenssoro E, Canales HS, Reina R, Saenz MG, Das Neves AV, et al. Clinical characteristics and outcomes of obstetric patients requiring ICU admission. Chest 2007;131(3):718-724. DOI: 10.1378/chest.06-2388.

3. Office of the Registrar General I. Special bulletin on maternal mortality in India 2016-18. Sample Regist Syst 2020;(July):1-4.

4. Gupta H, Gandotra N, Mahajan R. Profile of obstetric patients in intensive care unit: a retrospective study from a Tertiary Care Centre in North India. Indian J Crit Care Med 2021;25(4):388-391.

5. Rojas-Suarez J, Paternina-Caicedo AJ, Miranda J, Mendoza R, DueñasCastel C, Bourjeily G. Comparison of severity-of-illness scores in critically ill obstetric patients: a 6-year retrospective cohort. Crit Care Med 2014;42(5):1047-1054. DOI: 10.1097/ccm.0000000000000124.

6. Ashraf N, Mishra SK, Kundra P, Veena P, Soundaraghavan S, Habeebullah S. Obstetric patients requiring intensive care : a one year retrospective study in a tertiary care institute in India. Anesthesiol Res Pract 2014;2014:1-5. DOI: 10.1155/2014/789450.

7. Al-Suleiman SA, Qutub HO, Rahman J, Rahman MS. Obstetric admissions to the intensive care unit: a 12-year review. Arch Gynecol Obstet 2006;274(1):4-8. DOI: 10.1007/s00404-004-0721-z.

8. Jamal S, Mehta A, Goel N, Ahuja M, Afreen N, Malik S. Obstetrics ICU admissions: challenges faced at a tertiary referral centre. Int J Reprod Contracept Obstet Gynecol 2018;7(5):1840. DOI: 10.18203/2320-1770. ijrcog20181914.

9. Zwart JJ, Dupuis JRO, Richters A, Öry F, Van Roosmalen J. Obstetric intensive care unit admission: a 2-year nationwide population-based cohort study. Intensive Care Med 2010;36(2):256-263. DOI: 10.1007/ s00134-009-1707-x. 